\title{
EDITORIAL
}

\section{A Clinician's Guide to the Efficacy of EMDR Therapy}

\author{
Louise Maxfield \\ Ridgetown, Ontario, Canada
}

\begin{abstract}
Thirty years after its introduction in 1989, eye movement desensitization and reprocessing (EMDR) therapy has evolved to become a comprehensive psychotherapy, guided by Shapiro's adaptive information processing model. Her model views most mental health disorders as stemming from unprocessed earlier disturbing events. This understanding of the etiological role of trauma has opened the door for EMDR treatment of multiple types of presentations. There are now more than 44 randomized controlled trials that have investigated EMDR treatment of posttraumatic stress disorder (PTSD), early traumatic stress, and traumatized children. In addition, there are 28 randomized controlled trials which have evaluated its use with major depressive disorder, bipolar disorder, psychosis, anxiety disorders, obsessive compulsive disorder, substance use disorder, and pain. Seventy-five percent of these studies provided Shapiro's standardized procedure, while others tested modifications developed for specific populations. The focus of treatment varied across the studies, with various targets being processed to achieve good outcomes. The research demonstrates EMDR's effectiveness in reducing/eliminating PTSD and trauma-related symptoms, and in improving symptoms related to presenting problems and disorders. EMDR can be considered to have well-established efficacy for the treatment of PTSD. The emerging evidence for EMDR's efficacy with disorders other than PTSD must be considered preliminary and in need of replication conducted with randomized controlled trials using rigorous methodology. EMDR's position in various treatment guidelines is discussed, and the needs for future research are elaborated.
\end{abstract}

Keywords: eye movement desensitization and reprocessing (EMDR) therapy; efficacy; treatment outcome; review

$\mathbf{T}$ his special issue of the Journal of EMDR Practice and Research commemorates the 30th anniversary of eye movement desensitization and reprocessing (EMDR) therapy. It is titled A Clinician's Guide to the Efficacy of EMDR Therapy, and it provides brief narrative summaries of EMDR's efficacy for clients presenting with various diagnoses. Most of the articles in this issue are not comprehensive systematic reviews. Their goal is to provide short useful descriptions of EMDR's effects, and an overview of the growing body of evidence for its use with a range of diagnostic categories. Articles discuss EMDR's effects for adults with posttraumatic stress disorder (PTSD), early traumatic stress, complex PTSD, major depressive disorder, bipolar disorder, psychosis, anxiety disorders, obsessive compulsive disorder (OCD), substance use disorder, and pain, as well the treatment of traumatized children. There is also an article analyzing the differences in recommendations made by treatment guideline committees and an article outlining recommendations for future research with global implications. EMDR can be considered to have wellestablished efficacy for treatment of adults with PTSD. The emerging evidence for EMDR's efficacy with disorders other than PTSD must be considered preliminary and in need of replication conducted with randomized controlled trials (RCTs) using rigorous methodology.

\section{EMDR Therapy and Treatment Conceptualization}

EMDR therapy differs from other trauma-focused treatments that seek to decrease fear, reduce avoidant 
behaviors, or change problematic cognitions. Instead, EMDR therapy works on changing the memory. The established by-products of EMDR's memory transformation are reduction/elimination of PTSD diagnoses and presenting symptoms, negative affect, problematic behaviors, and erroneous cognitions. Other effects, reported anecdotally and in case studies, require rigorous research investigation.

The adaptive information processing (AIP) model states that most psychological disorders "are forged by earlier experiences that contain affects of 'helplessness,' 'hopelessness,' or any of the full spectrum of emotions that constitute a sense of self-denigration and lack of personal efficacy" (F. Shapiro, 2018, p. 5). The model conceptualizes most pathology as symptoms of unprocessed memories that are stored in statespecific form, and that are elicited when current situations activate the memory, allowing the negative affect and beliefs from the past to impact the present. The AIP model posits that individuals have an inherent information processing system that moves naturally towards health, growth, and learning. It also predicts the observed and measured effects of EMDR therapy, stating that when the past, present, and future aspects of the disturbing experience are processed with EMDR therapy, memory networks containing adaptive information are spontaneously accessed and integrated with the dysfunctional memories, leading to transformation and resolution.

\section{The Special Issue}

This special issue is conceptualized as a clinician's guide to the efficacy of EMDR therapy. It contains 11 short review articles, each evaluating the level of evidence for EMDR therapy within a diagnostic category. The issue also includes an analysis of the differences in international treatment guidelines (Dominguez \& Lee, 2019) and a call for future research with global implications (F. Shapiro, 2019).

Inclusion criteria for the review articles were a focus on (a) RCTs and (b) investigation of EMDR's effects with a specific diagnosis. Although some authors (Barron, Bourgaize, Lempertz, Swinden, \& Barker-Smith, 2019; Tapia, 2019) conducted systematic reviews, the overall goal of the issue was to provide brief useful summaries that would be helpful for therapists.

Some readers may wonder why the issue focused on findings from RCTs. There were several reasons for this. Although case studies and qualitative studies produce rich detailed information about the treatment of one or a few individuals, they cannot rule out that the possibility that their positive outcome was due to a selection bias, the passage of time, the therapeutic relationship, spontaneous remission, or other factors. RCTs are designed to control for these potential causes of improvement, and they allow for the conclusion that the treatment alone probably caused the positive results. Studies using a randomized controlled design are the primary, and often only, type of study examined by treatment guideline committees and in metaanalyses. RCTs are used to determine efficacy and to compare effect sizes and levels of evidence.

Readers may also wonder why inclusion criteria for this issue required studies with diagnosed participants (with exceptions of articles on recent trauma, pain, and children). While it can be argued that many clinicians work with clients whose presentation does not meet criteria for a diagnosis, individuals with diagnosed disorders tend to have more severe presentations and higher levels of impaired function. It is assumed that a treatment that is effective for those with the most severe symptoms should also be helpful for those whose presentation is less debilitating - but it cannot be assumed that a treatment effective for individuals with moderate symptoms will also remediate an incapacitating level of symptoms. In addition, many treatment guidelines and meta-analyses include only RCTs with diagnosed participants. Although some readers may be concerned that results from RCTs will not generalize to their clients with comorbid presentations, such a concern is misplaced, as the diagnosed participants in most studies have a wide range of comorbid symptoms.

\section{Trauma- and Stressor-Related Disorders}

The first year for publications of RCTs investigating treatments for PTSD was 1989. The author of one of those studies was Francine Shapiro, whose research introduced a new treatment called eye movement desensitization (F. Shapiro, 1989). Today, 30 years later, the treatment - now called EMDR therapyhas evolved to become a comprehensive psychotherapy that focuses on the full clinical picture, not just symptom reduction (F. Shapiro, 2018).

\section{Posttraumatic Stress Disorder}

As can be seen in Table 1, there are more than 44 RCTs that have investigated EMDR treatment for traumaand stressor-related disorders. EMDR therapy was initially developed as a PTSD treatment, and so it is not surprising that EMDR treatment has been primarily used for trauma presentations and that EMDR 
TABLE 1. Randomized Controlled Trials for Trauma- and Stress-Related Disorders

\begin{tabular}{|c|c|c|c|}
\hline Article in this Issue & Presenting Disorder & N RCTs & Protocols \\
\hline De Jongh et al. (2019) & PTSD in adults & $30+$ & Standard EMDR $(N=29+)$; E. Shapiro’s G-TEP $(N=1)$ \\
\hline \multirow[t]{2}{*}{ Barron et al. (2019) } & PTSD in children & 4 & $\begin{array}{l}\text { Beere \& De Roos's Dutch EMDR protocol for children } \\
\text { and adolescents }(N=2) \text {; Standard EMDR with Ahmed's } \\
\text { child-adjusted EMDR protocol }(N=1) \text {; Standard EMDR } \\
\text { with Tinker's adaptations }(N=1)\end{array}$ \\
\hline & $\begin{array}{l}\text { Traumatized children (no } \\
\text { diagnosis) }\end{array}$ & 3 & $\begin{array}{l}\text { Beere } \& \text { De Roos's Dutch EMDR protocol for children } \\
\text { and adolescents }(N=1) \text {; Standard EMDR with } \\
\text { Greenwald's adaptions }(N=2)\end{array}$ \\
\hline De Jongh et al. (2019) & Complex PTSD & 0 & NA \\
\hline $\begin{array}{l}\text { E. Shapiro \& Maxfield } \\
(2019)\end{array}$ & $\begin{array}{l}\text { Adults with early trauma } \\
\text { (no diagnosis) }\end{array}$ & 7 & $\begin{array}{l}\text { F. Shapiro's recent event protocol }(N=1) \text {; E. Shapiro \& } \\
\text { Laub's R-TEP }(N=3) \text {; Jarero et al.'s EMDR PRECI, and } \\
\text { PROPARA }(N=3)\end{array}$ \\
\hline
\end{tabular}

Note. G-TEP = group traumatic episode protocol; PRECI = protocol for recent critical incidents; PROPARA = protocol for paraprofessional use; PTSD = posttraumatic stress disorder; RCT $=$ randomized controlled trial; R-TEP $=$ Recent Traumatic Episode Protocol.

research has mainly focused on the remediation of this disorder. De Jongh, Amann, Hofmann, Lee, and Farrell (2019) report that there are more than 30 RCTs showing EMDR's strong clinical effects for adults diagnosed with PTSD, and 4 articles for children with ful1/ partial diagnosed PTSD. Results show that the provision of standard EMDR therapy significantly decreases or eliminates PTSD diagnosis and symptoms, with results maintained at follow-up. In their article, De Jongh, Amann, et al. summarize the 13 adult and 2 child RCTs that directly compared EMDR and traumafocused cognitive behavioral therapy (TF-CBT). They state that EMDR appears to produce outcomes similar to those of TF-CBT, and that there is a possibility that EMDR may be more efficient and more cost-effective. Concluding that the evidence base for EMDR therapy is robust and that it should be considered a first-choice treatment for PTSD, they discuss related treatment guidelines.

\section{Treatment Guidelines}

The evidence for EMDR treatment for PTSD appears to be solid, consistent, and well established, and the treatment guideline committee of the International Society for Traumatic Stress Studies gave EMDR a strong recommendation for adults and children with PTSD and a standard recommendation for early intervention (ISTSS Guidelines Committee, 2018). However, the treatment guideline committees for the American Psychological Association (APA, 2017) and the UK National Institute for Health and Care
Excellence (NICE, 2018) did not reach the same conclusions. Instead APA gave EMDR therapy only a conditional recommendation for the treatment of adult PTSD. NICE recommended EMDR only for civilian adults, and their recommendations for child treatment and recent events were conditional. These three guidelines were analyzed by Dominguez and Lee (2019), who determined the nature of, and reasons for, the major discrepancies. They concluded that the 2017 APA guidelines had the least validity, but noted that variations in methodology in EMDR research studies leaves the guidelines vulnerable to such evaluations. The treatment guidelines are also discussed in articles on PTSD (De Jongh et al., 2019), early intervention (E. Shapiro \& Maxfield, 2019), and traumatized children (Barron et al., 2019).

\section{Recent Trauma}

E. Shapiro and Maxfield (2019) examine the results in seven RCTs providing individual EMDR early intervention within 3 months of a traumatic event. There was substantial heterogeneity among the studies, which used different populations, different time periods posttrauma, and different protocols. See Table 1. Results showed significant reductions in symptoms of traumatic stress with results maintained at followup, but methodological limitations decrease the level of confidence in the findings. Research is still needed to investigate whether EMDR early intervention prevents the development of PTSD or increases resilience. It should be noted that there are no RCTs investigating the treatment of recent trauma with children, and 
none evaluating group treatment within the 3-month post-trauma period.

\section{Traumatized Children}

The treatment of children and adolescents is poorly represented in the EMDR literature. As noted in the systematic review by Barron et al. (2019), there are only seven RCTs investigating the treatment of traumatized children, four of these with children diagnosed with full/partial PTSD. These studies all describe using F. Shapiro's standard procedures, with various types of accommodations for the children's developmental stage. See Table 1. The authors reported that EMDR was efficacious in reducing children's PTSD symptoms, with results maintained at follow-up. The authors assert that EMDR should be considered an efficacious treatment for children with PTSD, and their conclusion is also that of the ISTSS (2018) treatment guidelines, which recommend EMDR therapy for children and adolescents.

\section{Complex PTSD}

Although complex PTSD is a fairly common clinical presentation, EMDR treatment for this population has yet to be directly investigated in any RCT studies. De Jongh et al. (2019) summarize indirect research support that provides preliminary indications that EMDR can effectively be used for clients with a history of early childhood interpersonal trauma, who suffer from complex PTSD symptoms. The article further notes that there appears to be little empirical evidence that treatment requires a stabilization phase prior to trauma therapy.

\section{Beyond PTSD}

Many studies that investigated EMDR treatment of PTSD also examined its effects on comorbid symptoms of depression and anxiety, often reporting significant decreases in such symptoms. A meta-analysis of 26 PTSD studies that evaluated EMDR treatment reported improvements in comorbid symptoms of anxiety and depression, with moderate effect sizes (Chen et al., 2014). Further, a meta-analysis by Ho and Lee (2012) compared the effects of TF-CBT and EMDR on eight PTSD studies. They reported that while there was no difference in PTSD outcomes, EMDR had a significant advantage over TF-CBT in reducing comorbid depressive symptoms, with a moderate effect size.
As stated earlier in this article, the AIP model views most pathology as symptoms of unprocessed disturbing memories (F. Shapiro, 2018). In other words, disorders that are not typically viewed as trauma-based are seen through the EMDR lens as having their roots in earlier traumatic events. This understanding of the etiological role of trauma has opened the door for EMDR treatment of multiple types of presentations. F. Shapiro notes that treatment must address the specific symptoms and characteristics of each diagnosis, and points out the importance of a comprehensive treatment plan to address the full clinical picture.

There are now 28 RCTs that have investigated its use for depression, bipolar disorder, psychosis, anxiety disorders, OCD, substance use disorder, and pain. See Table 2. In $75 \%$ of these studies, EMDR treatment was provided using F. Shapiro's (2018) standard eight-phase, three-pronged EMDR protocol, which processed the events related to the onset of disorder or its distressing aspects. The modified procedures used in the other studies include types of targets, methods of target selection, sequential order of processing, and the addition of other elements, such as CBT techniques. For example, in the addiction-focused treatment model, the treatment target is cravings, rather than memories.

\section{Depression}

Malandrone, Carletto, Hofmann, Hase, and Ostacoli (2019) outline the seven RCTs that have shown EMDR to be effective in the treatment of depression. The studies found good outcomes on self-report measures, but no diagnostic assessments were done after treatment. There is substantial heterogeneity among the studies, which used different populations (e.g., major depression, treatment resistant depression, recurrent depression) and different protocols: standard EMDR, modified EMDR, and Hofmann et al.'s (2016) DeprEND. See Table 2. Results showed that EMDR's effects were significantly better than wait list and similar to those of CBT, and that EMDR appeared to provide a benefit as an adjunctive treatment. The research for depression is still in its early stages, but is ongoing at various international sites.

\section{Bipolar Disorder}

EMDR research is also currently being conducted for bipolar disorder. In this issue, Valiente-Gomez et al. (2019) describe the nature and etiology of bipolar disorder, explaining why and how EMDR treatment 
TABLE 2. Randomized Controlled Trials for Disorders Beyond PTSD

\begin{tabular}{|c|c|c|c|}
\hline Article in this Issue & $\begin{array}{l}\text { Presenting } \\
\text { Disorder }\end{array}$ & $\begin{array}{l}\text { Number of } \\
\text { RCTs }\end{array}$ & Protocols \\
\hline $\begin{array}{l}\text { Malandrone et al. } \\
\text { (2019) }\end{array}$ & Depression & 7 & $\begin{array}{l}\text { Standard EMDR }(n=4) \text {; Standard EMDR with Gauhar's } \\
\text { adaptation re target selection }(n=1) \text {; EMDR Plus TAU } \\
\text { Hofmann et al.'s DeprEND protocol }(n=2)\end{array}$ \\
\hline $\begin{array}{l}\text { Valiente-Gómez et al. } \\
\text { (2019) }\end{array}$ & Bipolar & 1 & Standard EMDR $(n=1)$ \\
\hline De Bont et al. (2019) & Psychosis & 3 & Standard EMDR $(n=3)$ \\
\hline \multirow[t]{3}{*}{$\begin{array}{l}\text { Faretta and Dal Farra } \\
(2019)\end{array}$} & $\begin{array}{l}\text { Panic disorder } \\
\text { w/wo } \\
\text { agoraphobia }\end{array}$ & 3 & Standard EMDR $(n=3)$ \\
\hline & Simple phobia & 2 & $\begin{array}{l}\text { Standard EMDR }(n=1) \text {; Triscari’s EMDR + CBT } \\
\text { protocol }(n=1)\end{array}$ \\
\hline & $\begin{array}{l}\text { Anxiety disorder, } \\
\text { low self-esteem }\end{array}$ & 1 & Standard EMDR $(n=1)$ focused on self-esteem \\
\hline Böhm (2019) & OCD & 2 & Standard EMDR $(n=1) ;$ Marr's OCD protocol $(n=1)$ \\
\hline Tapia (2019) & $\begin{array}{l}\text { Substance use } \\
\text { disorder }\end{array}$ & 3 & $\begin{array}{l}\text { Standard EMDR }(n=1) \text {; Hases' addiction-focused EMDR } \\
(n=1) \text {; Eye movements only }(n=1)\end{array}$ \\
\hline $\begin{array}{l}\text { Tesarz, Wicking, } \\
\text { Bernardy, and Seidler } \\
\text { (2019) }\end{array}$ & Pain & 6 & $\begin{array}{l}\text { Standard EMDR }(n=5) \text {; Marcus's integrated EMDR for } \\
\text { migraine }(n=1)\end{array}$ \\
\hline
\end{tabular}

Note. $\mathrm{CBT}=$ cognitive behavioral therapy $\mathrm{OCD}=$ obsessive compulsive disorder; $\mathrm{PTSD}=$ posttraumatic stress disorder. 21 standard; 7 unique protocols.

might reduce subsequent mood episodes and affective and trauma-related symptoms, as well as improving cognitive and social function. Only one RCT has been published, and although the results were promising, they were not maintained at follow-up. The researchers have now developed a more comprehensive EMDR protocol to address the various symptoms and clinical presentations of this complex disorder. There is also a case study that investigated neurobiological changes for a patient with bipolar disorder, pre-post EMDR treatment, finding normalization of the default mode network.

\section{Psychosis}

EMDR has preliminary evidence of effectiveness in the treatment of patients with psychosis. In this issue, De Bont, De Jongh, and Van den Berg (2019) discuss the outdated paradigm that considered trauma-focused treatment to be contraindicated for patients with psychosis, due to a concern that such therapy would exacerbate symptoms. However, De Bont et al. report that research has shown that there is no basis for this restriction, and that the reduction of trauma-related symptoms can be very helpful for this populations. The effects of EMDR treatment are not limited to a decrease in PTSD symptoms, but also appear to reduce some psychotic symptoms, such as delusional and paranoid thoughts, with improvement in quality-of-life measures. A cost analysis found substantial financial savings over the 6 months posttreatment for patients treated with EMDR. De Bont et al. discuss other EMDR-related research that has focused directly on reducing psychotic symptoms.

\section{Anxiety Disorders}

Faretta and Dal Farra (2019) discuss the importance of using the AIP model to conceptualize treatment for individuals with anxiety disorders. They summarize three RCTs for panic disorder with/ without agoraphobia, two RCTs for phobias, and one related to self-esteem in patients with anxiety disorders. They also discuss other case and quasi-experimental studies. There is great heterogeneity within the anxiety disorder studies, even within specific diagnostic categories. For example, two early studies on EMDR treatment for panic disorder produced mixed outcomes. F. Shapiro (2018) noted possible problems such as lack of treatment 
fidelity, insufficient treatment time, and procedural restrictions, and said the treatment should have addressed related childhood experiences. More positive results were produced in a recent RCT for participants with panic disorder, which processed past memories, current triggers, and future templates, to successfully address emotional responses and avoidant behaviors, producing the same outcomes as an established CBT procedure. While two RCTs on simple phobias for flying and dental procedures addressed related traumatic memories and showed successful treatment with EMDR therapy, an RCT that attempted to improve selfesteem found that EMDR did not produce optimal effects. In this study, EMDR treatment did not target memories unrelated to self-esteem, and processing was hampered by associations to untreated traumas.

\section{Obsessive Compulsive Disorder}

The treatment for OCD has been evaluated in various case studies and two RCTs (Böhm, 2019). One RCT compared a modified EMDR protocol to an established exposure response and prevention therapy and found no differences between the two. The other RCT provided the standard EMDR protocol and resulted in a better outcome than medication. Case studies have used other treatment protocols, such as integrating EMDR and CBT strategies. There has been little consistency in this field. Böhm discusses treatment conceptualization and strategies. See Table 2.

\section{Substance Use Disorder}

A systematic review for EMDR treatment of substance use disorders was conducted by Tapia (2019) in this issue. She summarizes the evidence for traumafocused EMDR treatment, addiction-focused EMDR treatment, and combined trauma- and addictionfocused treatment. One RCT evaluated traumafocused EMDR treatment, and two RCTs evaluated addiction-focused EMDR. The body of research indicates that although trauma-focused EMDR treatment may effectively reduce PTSD symptoms, it seems to have little impact on substance use. The addictionfocused EMDR may reduce craving and improve remission, but does not appear to impact traumatic memories. Future research is investigating combined trauma- and addiction-focused EMDR therapy for patients with substance use disorder. An important result of this work has been the finding that traumafocused treatments, such as EMDR, can be safely provided to individuals with active substance use disorder. The old paradigm believed that trauma therapy was contraindicated for patients with active substance use, anticipating worsening of the addiction and little positive effect from treatment; however, research has found that the treatment of trauma is beneficial even for those without sobriety.

\section{Pain}

EMDR's effectiveness in the treatment of pain has been noted for many years, especially with regard to the treatment of phantom-limb pain. As noted in this issue, pain has become a new exciting area for the expansion of EMDR treatment (Tesarz et al., 2019). There are now six RCTs, all examining treatment of different kinds of pain - from phantom limb pain, to chronic pain, to acute post-surgery pain. With one exception, all the RCTs used F. Shapiro's standard procedures. Targets included pain experience, negative thoughts or images related to pain, memories of pain's onset, and unrelated traumatic memories. Results show decreased pain intensity, with large effect sizes, and where measured, results were maintained at follow-up. These impressive findings should be interpreted cautiously, given the methodological limitations in the research. Tesarz et al. provide a comprehensive overview of the studies and discuss the possible mechanisms for these effects.

\section{Recommendations for Clinicians}

With more than 70 RCTs examining the use of EMDR therapy, clinicians can have confidence that the treatment is effective and safe in the treatment of traumatic stress. It has been used worldwide, for thousands of participants presenting with a wide range of problems and disorders. The broader application of EMDR to various other disorders is an exciting development-however, most non-PTSD research is still in its infancy. There are many protocols and modified procedures, with resulting heterogeneity and lack of certainty whether the results of any modified nonPTSD protocol will generalize to populations outside of the participants in the study. Therefore, therapists considering using EMDR interventions for other disorders should be conservative, consider that the modified procedure is still an experimental application, and carefully monitor the client's response to treatment. Keep in mind as well the admonition of F. Shapiro (2018) that the goal is to treat the full clinical picture. 


\section{Future Research}

\section{Investigations of Efficacy}

Research is needed to investigate EMDR's efficacy for each of the disorders discussed in this article. Although the evidence for PTSD is robust, rigorous RCTs remain essential for its continued recognition as efficacious. The evidence for all other disorders can be considered only preliminary. It is important that research follow established methodological guidelines such as those of the Consort 2010 guidelines for randomized research (http://www.consortstatement.org/).

As noted in our methodological meta-analysis (Maxfield \& Hyer, 2002), many older studies had incomplete randomization and lacked blind assessments, multimodal assessments, and fidelity assessments. These limitations are still apparent in some more recent articles, and in others that do not provide necessary information regarding design, participant selection, randomization procedures, assessment procedures, and therapeutic details. Such articles are at risk of being rated as "biased" and discarded by treatment guideline committees and cannot contribute to our understanding of EMDR's efficacy. Information on statistical, procedural, and therapeutic details is essential for other researchers and clinicians who wish not only to evaluate the quality of the data, but to understand what was done in the study and how it might apply to their own work.

\section{Expanding Outcome Evaluations}

Most outcome research in EMDR treatment has focused on self-report measurements of PTSD and other presenting symptoms. Multimodal evaluations are strongly encouraged. In addition to self-report inventories, researchers should use interview assessments, such as the Clinician Administered PTSD Scale, in order to make a clinical diagnosis and to gain a more complete picture of the individual client's pathology. Other useful tools are behavioral measures, such as daily counts of frequency and/or intensity of behaviors (e.g., angry outbursts, avoidance, pain) to evaluate change over time. Cost analyses are another important evaluation to consider.

Outcomes should also move from simply assessing symptom reduction to looking at some of the more transformative changes seen in EMDR therapy. Positive outcomes such as resilience and growth must be evaluated. Other effects that have been reported anecdotally and in case studies require rigorous research investigation in future RCTs. These include reversals of trauma's devastating effects on the individual's neurobiology, physical health, cognitive function, affect regulation, interpersonal relationships, and selfconcept.

\section{Investigating Treatment Process}

The selection of treatment targets is an important area for future research, not only with regard to cessation of suffering, but also for the insight that such applications may provide into the mechanisms of EMDR's effects and the relevance for clinicians seeking to translate research into practice. Questions posed in this issue by Tesarz et al. (2019) about pain disorders apply globally to all disorders discussed in this article:

Which therapy targets should be focused on ...? In which order should the targets be processed? For which group of ... patients is EMDR more suitable and for which not? Which form of EMDR protocol should be used? The current state of the studies does not provide a clear answer to these questions. (p. 342)..... However, it can be assumed that different patient groups will benefit from different approaches and it will be the challenge of future studies to test which approach is best for which patient group.

(p. 343)

\section{Prevention}

The AIP model (F. Shapiro, 2018) predicts that the treatment of traumatic memories will not only remediate presenting symptoms, it will also prevent future occurrences and the trauma-related development of various types of disorders and physical health problems. Long-term research, comparing treated and untreated individuals, is needed to determine the longterm benefits of EMDR therapy.

\section{Global Implications}

F. Shapiro (2019) argues that EMDR clinicians and researchers have a global responsibility to investigate the value of various treatment options in the parts of the world where there are few mental health resources. She advocated that we work together to find ways to bring the healing power of EMDR therapy to everyone-especially to those in greatest need. 


\section{References}

American Psychological Association. (2017). Clinical practice guideline for the treatment of posttraumatic stress disorder (PTSD) in adults. Retrieved from https: / / www.apa.org/ptsd-guideline/ptsd.pdf

Böhm, K. R. (2019). EMDR's efficacy for obsessive compulsive disorder (OCD. Journal of EMDR Practice and Research, 13(4), 333-336. doi:10.1891/1933-3196.13.4.333

Barron, I. G., Bourgaize, C., Lempertz, D., Swinden, C., \& Barker-Smith, S. (2019). Eye movement desensitization reprocessing for children with posttraumatic stress disorder: A systematic narrative review. Journal of EMDR Practice and Research, 13(4), 270-283. doi:10.1891/19333196.13.4.270

Chen, Y. R., Hung, K. W., Tsai, J. C., Chu, H., Chung, M. H., Chen, S. R., . . Chou, K. R. (2014). Efficacy of eyemovement desensitization and reprocessing for patients with posttraumatic-stress disorder: A meta-analysis of randomized controlled trials. PLOS ONE, 9(8), e103676. doi:10.1371/journal.pone.0103676

De Bont, P., De Jongh, A., \& Van den Berg, D. (2019). Psychosis: An emerging field for EMDR research and therapy. Journal of EMDR Practice and Research, 13(4), 313-324. doi:10.1891/1933-3196.13.4.313

De Jongh, A., Bicanic, I., Matthijssen, S., Amann, B. L., Hofmann, A., Farrell, D., . . Maxfield, L. (2019). The current status of EMDR therapy involving the treatment of complex posttraumatic stress disorder. Journal of EMDR Practice and Research, 13(4), 261-269. doi:10.1891/19333196.13.4.284

Dominguez, S., \& Lee, C. W. (2019). Differences in international guidelines regarding EMDR for PTSD: Why they diverge and suggestions for future research. Journal of EMDR Practice and Research, 13(4), 247-260. doi:10.1891/1933-3196.13.4.247

Faretta, E., \& Dal Farra, M. (2019). Efficacy of EMDR therapy for anxiety disorders. Journal of EMDR Practice and Research, 13(4), 325-332. doi:10.1891/1933-3196.13.4.325

Ho, M. S. K., \& Lee, C. W. (2012). Cognitive behaviour therapy versus eye movement desensitization and reprocessing for post-traumatic disorder-Is it all in the homework then? European Review of Applied Psychology, 62, 253-260. doi:10.1016/j.erap.2012.08.001

Hofmann, A., Hase, M., Liebermann, P., Ostacoli, L., Lehnung, M., Ebner, F., . . . Tumani, V. (2016). DeprEnd(C-EMDR therapy protocol for the treatment of depressive disorders. In M. Luber (Ed.), Eye movement desensitization and reprocessing (EMDR) therapy scripted protocols and summary sheets: Treating anxiety, obsessivecompulsive, and mood-related conditions (pp. 289-311). New York, NY: Springer Publishing.
ISTSS Guidelines Committee. (2018). Posttraumatic stress disorder prevention and treatment guidelines methodology and recommendations. Oakbrook Terrace, IL: Author. Retrieved from http://www.istss.org/ treating-trauma/new-istss-prevention-and-treatmentguidelines.aspx

Malandrone, F., Carletto, S., Hofmann, A., Hase, M., \& Ostacoli, L. (2019). A brief narrative summary of randomized controlled trials investigating EMDR treatment of patients with depression. Journal of EMDR Practice and Research, 13(4), 302-306. doi:10.1891/1933-3196.13.4.302

Maxfield, L., \& Hyer, L. (2002). The relationship between efficacy and methodology in studies investigating EMDR treatment of PTSD. Journal of Clinical Psychology, 58, 23-41. doi:10.1002/jclp.1127

National Institute for Health and Care Excellence. (2018). Post-traumatic stress disorder. Evidence reviews on care pathways for adults, children and young people with PTSD. Retrieved from https://www.nice.org. uk/guidance/ng116

Shapiro, F. (1989). Efficacy of the eye movement desensitization procedure in the treatment of traumatic memories. Journal of Traumatic Stress, 2, 199-223. doi: $10.1002 /$ jts. 2490020207

Shapiro, F. (2018). Eye movement desensitization and reprocessing (EMDR): Basic principles, protocols, and procedures (3rd ed.). New York, NY: Guilford Press.

Shapiro, E., \& Maxfield, L. (2019). The efficacy of EMDR early interventions. Journal of EMDR Practice and Research, 13(4), 291-302. doi:10.1891/1933-3196.13.4.219

Shapiro, F. (2019). Future research: Global implications. Journal of EMDR Practice and Research, 13(4), 354-360. doi:10.1891/1933-3196.13.4.354

Tapia, G. (2019). EMDR for treating substance use disorders. Journal of EMDR Practice and Research, 13(4), 345353. doi:10.1891/1933-3196.13.4.345

Tesarz, J., Wicking, M., Bernardy, K., \& Seidler, G. H. (2019). EMDR therapy's efficacy in the treatment of pain. Journal of EMDR Practice and Research, 13(4), 337-344. doi:10.1891/1933-3196.13.4.337

Valiente-Gómez, A., Moreno-Alcázar, A., Gardoki-Souto, I., Masferrer, C., Porta, S., Royuela, O., \& . . Amann, B. L. (2019). The state of art of EMDR in bipolar disorder. Journal of EMDR Practice and Research, 13(4), 307-312. doi:10.1891/1933-3196.13.4.307

Disclosure. L. M. receives income from a published book on EMDR therapy and is the editor-in-chief of this journal.

Correspondence regarding this article should be directed to Louise Maxfield, PO Box 1165, Ridgetown, ON N0P2C0. Email: Dr.Maxfield@outlook.com 